\title{
THE GENERALIZED PARTIAL CORRESPONDENCE PRINCIPLE IN LINEAR VISCOELASTICITY*
}

\author{
By \\ G. A. C. GRAHAM ${ }^{1}$ (Dublin Institute for Advanced Studies, Ireland)
}

AND

J. M. GOLDEN ${ }^{2}$ (National Institute for Physical Planning and Construction Research, Dublin, Ireland)

Summary. A result, referred to as the generalized partial correspondence principle, is proved for noninertial viscoelastic boundary value problems. This states that if $B_{u}^{(i)}(t), B_{\sigma}^{(i)}(t)$ are the complementary regions of the boundary of a viscoelastic medium with a unique Poisson's ratio, on which displacements $u_{i}(\mathbf{r}, t)$ and stresses $\sigma_{i j}(\mathbf{r}, t) n_{j}$ are specified, respectively, then if $B_{\sigma}^{(i)}\left(t^{\prime}\right) \subseteq B_{\sigma}^{(i)}(t)$ for all $t^{\prime} \leq t$, the stresses satisfying this mixed boundary value problem at time $t$ are the stresses for the elastic problem with the same boundary regions and the same specified stresses while known functions take the place of the specified displacements. It is noteworthy that $B_{\sigma}^{(i)}\left(t^{\prime}\right)$, $t^{\prime} \leq t$, is not required to be monotonic increasing.

Similarly, if $B_{u}^{(i)}(t)$ has the property $B_{u}^{(i)}\left(t^{\prime}\right) \subseteq B_{u}(t), t^{\prime} \leq t$, then the above statement holds but with stresses and displacements interchanged.

In certain cases, it is not necessary to assume that the material has a unique Poisson's ratio, though in these cases the statement of the principle is altered and though somewhat restricted still has a useful range of application.

The principle is applied to obtain certain nontrivial results of possible experimental interest for the normal contact problem, where the loading history is restricted only in the manner specified above.

1. Introduction. The original or classical form of the correspondence principle provided a powerful tool for solving mixed viscoelastic boundary value problems where the boundary regions are not time-dependent. This principle is discussed, for example, in [1], where references to the original papers are given. Later [2, 3, 4], extended forms of this principle were given which covered situations where the boundary regions are increasing or decreasing monotonically. This topic is discussed in more general terms in [5], which also addresses the situation where the body occupies an ablating region.

*Received June 26, 1987.

'On leave from: Department of Mathematics and Statistics, Simon Fraser University, Burnaby, B.C., Canada V5A 1 S6.

${ }^{2}$ Research Associate, Dublin Institute for Advanced Studies, 10 Burlington Road, Dublin 4, Ireland.

(C) 1988 Brown University 
In this paper, we present a generalization of the correspondence principle which applies to a far more general range of situations, but does not privide complete information on the solution. We will refer to it as the generalized partial correspondence principle to distinguish it from the principle developed in [2-5], which we refer to as the extended correspondence principle.

In Sec. 2 we prove the result under the assumption that the material has a unique Poisson's ratio. In Sec. 3 it is shown that the restriction on Poisson's ratio for the material is not always necessary. In Sec. 4 an interesting example is worked out. Some concluding observations are presented in Sec. 5.

2. Material with unique Poisson's ratio. The approach used is a generalization of that developed in [6]. Temperature variations are ignored, however. Also, a different notation is adopted. The constitutive and dynamical equations are written in the form (see Golden and Graham [7])

$$
\begin{aligned}
\sigma_{i j}(\mathbf{r}, t) & =2 \int_{-\infty}^{t} d t^{\prime} \mu\left(t-t^{\prime}\right) \varepsilon_{i j}\left(\mathbf{r}, t^{\prime}\right)+\delta_{i j} \int_{-\infty}^{t} d t^{\prime} \lambda\left(t-t^{\prime}\right) \varepsilon_{k k}\left(\mathbf{r}, t^{\prime}\right), \\
\sigma_{i j, j}(\mathbf{r}, t) & =0, \quad i=1,2,3,
\end{aligned}
$$

where the summation convention is in force and inertial effects are neglected. The quantities $\sigma_{i j}(\mathbf{r}, t)$ and $\varepsilon_{i j}(\mathbf{r}, t)$ are the stress and strain Cartesian tensor components, respectively, at position $\mathbf{r}=(x, y, z)$ and time $t$. The strain tensor is related to the displacements $u_{i}(\mathbf{r}, t)$ by the usual formulas:

$$
2 \varepsilon_{i j}(\mathbf{r}, t)=u_{i, j}(\mathbf{r}, t)+u_{j, i}(\mathbf{r}, t) .
$$

The kernels of the hereditary integrals in (2.1), namely $\mu(t)$ and $\lambda(t)$, are related to the relaxation functions for shear and bulk deformation. We have

$$
\mu(t)=G_{1}(0) \delta(t)+\dot{G}_{1}(t) H(t),
$$

where $G_{1}(t)$ is the shear relaxation function for the material, $H(t)$ is the Heaviside step function, and $\delta(t)$ is the singular delta function. There is a similar relation between $\lambda(t)+2 \mu(t) / 3$ and the bulk relaxation function. Both $\lambda(t)$ and $\mu(t)$ are zero for negative $t$. If the material has a unique Poisson's ratio $\nu$ then

$$
\lambda(t)=\frac{2 \nu}{1-2 \nu} \mu(t) .
$$

If (2.4) is valid, then by defining the pseudodisplacements

$$
v_{i}(\mathbf{r}, t)=\int_{-\infty}^{t} d t^{\prime} \mu\left(t-t^{\prime}\right) u_{i}\left(\mathbf{r}, t^{\prime}\right)
$$

and taking account of (2.2), it is clear that (2.1) has the form of the elastic equations for a material with shear modulus unity and Poisson's ratio $\nu$ if the displacements $u_{i}(\mathbf{r}, t)$ are replaced by $v_{i}(\mathbf{r}, t)$.

Consider the viscoelastic boundary value problem

$$
\begin{aligned}
u_{i}(\mathbf{r}, t) & =d_{i}(\mathbf{r}, t), & & \mathbf{r} \in B_{u}^{(i)}(t), \\
\sigma_{i j}(\mathbf{r}, t) n_{j}(\mathbf{r}) & =c_{i}(\mathbf{r}, t), & & \mathbf{r} \in B_{\sigma}^{(i)}(t),
\end{aligned}
$$


where $B_{u}^{(i)}(t)$ and $B_{\sigma}^{(i)}(t)$ are disjoint regions of the boundary $B$ of the medium such that

$$
B=B_{u}^{(i)}(t) \cup B_{\sigma}^{(i)}(t)
$$

and where $d_{i}(\mathbf{r}, t)$ and $c_{i}(\mathbf{r}, t)$ are specified functions. Now let $B_{\sigma}^{(i)}\left(t^{\prime}\right) \subseteq B_{\sigma}^{(i)}(t)$ for all $t^{\prime} \leq t$. Otherwise, the history of $B_{\sigma}^{(i)}\left(t^{\prime}\right)$ is arbitrary. It need not be monotonic, for example. This implies that $B_{u}^{(i)}(t)$ is contained in all $B_{u}^{(i)}\left(t^{\prime}\right), t^{\prime} \leq t$, so that

$$
v_{i}(\mathbf{r}, t)=\int_{-\infty}^{t} d t^{\prime} \mu\left(t-t^{\prime}\right) d_{i}\left(\mathbf{r}, t^{\prime}\right), \quad \mathbf{r} \in B_{u}^{(i)}(t),
$$

is known, since if $\mathbf{r}$ is in the region $B_{u}^{(i)}(t)$ it follows that it is in all regions $B_{u}^{(i)}\left(t^{\prime}\right)$, $t^{\prime} \leq t$. Therefore, the viscoelastic problem can be reduced to an elastic problem with the $v_{i}(\mathbf{r}, t)$ playing the part of displacements, where on the boundary region $B_{u}^{(i)}(t)$, $v_{i}(\mathbf{r}, t)$ is given by $(2.8)$ and on $B_{\sigma}^{(i)}(t), \sigma_{i j}(\mathbf{r}, t) n_{j}$ is given by the second equation of (2.6). The dependence on $\mu(t)$ comes in only through the boundary value of $v_{i}(\mathbf{r}, t)$ given by (2.8). Thus, $\sigma_{i j}(\mathbf{r}, t)$ and $v_{i}(\mathbf{r}, t)$ at any point in the medium are given by the elastic solution for shear modulus unity and Poisson's ratio $\nu$, and boundary values specified as above. Therefore $\sigma_{i j}(\mathbf{r}, t)$ is known everywhere, if the elastic problem can be solved for the boundary regions $B_{u}^{(i)}(t), B_{\sigma}^{(i)}(t)$. In particular, if the $d_{i}\left(\mathbf{r}, t^{\prime}\right)$ vanish for all $t^{\prime} \leq t$, the stresses at time $t$ are given by the corresponding elastic stresses and can at most depend on $\nu$. This situation occurs in the case of certain crack problems.

The pseudodisplacements $v_{i}(\mathbf{r}, t)$ are also known. However, in general, the displacements cannot be deduced without a further assumption on $B_{u}^{(i)}\left(t^{\prime}\right)$, namely that it is stationary or monotonically decreasing for all $t^{\prime} \leq t$. This latter assumption brings us back to the extended correspondence principle as enunciated in the somewhat special case considered in [6]. In the more general case considered here, nothing can be said about the displacements.

Similarly, if $B_{u}^{(i)}\left(t^{\prime}\right) \subseteq B_{u}^{(i)}(t)$, the displacements are given in terms of the specified displacements $d_{i}(\mathbf{r}, t), \mathbf{r} \in B_{u}^{(i)}(t)$, and the known functions

$$
q_{i}(\mathbf{r}, t)=\int_{-\infty}^{t} d t^{\prime} \gamma\left(t-t^{\prime}\right) \sigma_{i j}\left(\mathbf{r}, t^{\prime}\right) n_{j}(\mathbf{r}) ; \quad \mathbf{r} \in B_{\sigma}^{(i)},
$$

where $\gamma(t)$ is the inverse of $\mu(t)$ in the sense that

$$
\int_{0}^{t} d t^{\prime} \gamma\left(t-t^{\prime}\right) \mu\left(t^{\prime}\right)=\int_{0}^{t} d t^{\prime} \mu\left(t-t^{\prime}\right) \gamma\left(t^{\prime}\right)=\delta(t)
$$

It is related to the shear creep function of the medium by an equation similar to (2.3). The statement prior to (2.9) may be demonstrated by transferring the hereditary integral on to the stresses in (2.1) to obtain

$$
\frac{1}{2} Q_{i j}(\mathbf{r}, t)=\varepsilon_{i j}(\mathbf{r}, t)+\frac{\nu}{1-2 \nu} \varepsilon_{k k}(\mathbf{r}, t) \delta_{i j},
$$

which has the elastic form in terms of the pseudostresses

$$
Q_{i j}(\mathbf{r}, t)=\int_{-\infty}^{t} d t^{\prime} \gamma\left(t-t^{\prime}\right) \sigma_{i j}\left(\mathbf{r}, t^{\prime}\right)
$$


If the specified stresses on $B_{\sigma}^{(i)}(t)$ are always zero then the $q_{i}(\mathbf{r}, t)$ are zero and the displacements are given by the elastic formula, which can depend at most on Poisson's ratio $\nu$. This is the case for certain contact problems.

The considerations of this section generalize readily to a nonisotropic material for which all the relaxation functions are proportional to each other, and to linearized nonisothermal theories. Also, nonvanishing body force fields may be incorporated.

3. Materials with two relaxation functions. The assumption that the material has a unique Poisson's ratio is not always necessary. It is difficult to give a general characterization of the class of problems for which the assumption is redundant. However, in this section we will isolate one problem class of this kind, in the sense that a restricted statement of the principle is possible in such cases.

The viscoelastic Papkovich-Neuber representation was given by Gurtin and Sternberg [8]. We write it in the form $\left(x_{1}, x_{2}, x_{3}=x, y, z\right)$

$$
2 \int_{-\infty}^{t} d t^{\prime} \mu\left(t-t^{\prime}\right) u_{i}\left(\mathbf{r}, t^{\prime}\right)=\int_{-\infty}^{t} d t^{\prime} \kappa\left(t-t^{\prime}\right) \phi_{i}\left(\mathbf{r}, t^{\prime}\right)-x_{j} \phi_{j, i}(\mathbf{r}, t)-\phi_{0, i}(\mathbf{r}, t),
$$

where $\phi_{i}, i=0,1,2,3$, are harmonic functions and $\kappa(t)$ is defined by the fact that its Fourier transform

$$
\hat{\kappa}(\omega)=\int_{-\infty}^{\infty} d t \kappa(t) e^{-i \omega t}
$$

is given by

$$
\hat{\kappa}(\omega)=3-4 \hat{\nu}(\omega), \quad \hat{\nu}(\omega)=\frac{\hat{\lambda}(\omega)}{2(\hat{\lambda}(\omega)+\hat{\mu}(\omega))},
$$

where $\hat{\mu}(\omega)$ is the complex modulus for shear and $\hat{\lambda}(\omega)+2 \hat{\mu}(\omega) / 3$ is the bulk complex modulus. Thus $\hat{\nu}(\omega)$ is a generalized Poisson's ratio of the material. The function $\kappa(t)$ has the causal property that it vanishes for $t<0$ [9]. This applies also to the inverse transform of $\hat{\nu}(\omega)$, namely $\nu(t)$.

Green and Zerna [10] consider for elastic materials a particular class of problems characterized by the fact that the shearing stress vanishes at all points in a plane, taken to be the $z=0$ plane. We will translate their observations so that they apply to a viscoelastic material. This class of problems can be solved by choosing $\phi_{1}=\phi_{2}=0$ and putting

$$
\begin{aligned}
& \phi_{3}=(-) \frac{\partial \phi(\mathbf{r}, t)}{\partial z}, \\
& \phi_{0}=(-) \int_{-\infty}^{t} d t^{\prime} \kappa_{1}\left(t-t^{\prime}\right) \phi\left(\mathbf{r}, t^{\prime}\right),
\end{aligned}
$$

where $\phi(\mathbf{r}, t)$ is a harmonic function and $\kappa_{1}(t)$ is defined by

$$
\hat{\kappa}_{1}(\omega)=\frac{1}{2}(\hat{\kappa}(\omega)-1)=1-2 \hat{\nu}(\omega) .
$$


The displacements are related to $\phi(\mathbf{r}, t)$ by

$$
\begin{aligned}
2 v_{x}(\mathbf{r}, t) & =2 \int_{-\infty}^{t} d t^{\prime} \mu\left(t-t^{\prime}\right) u_{x}\left(\mathbf{r}, t^{\prime}\right) \\
& =z \frac{\partial^{2} \phi}{\partial x \partial z}(\mathbf{r}, t)+\int_{-\infty}^{t} d t^{\prime} \kappa_{1}\left(t-t^{\prime}\right) \frac{\partial \phi}{\partial x}\left(\mathbf{r}, t^{\prime}\right), \\
2 v_{y}(\mathbf{r}, t) & =z \frac{\partial^{2} \phi(\mathbf{r}, t)}{\partial y \partial z}+\int_{-\infty}^{t} d t^{\prime} \kappa_{1}\left(t-t^{\prime}\right) \frac{\partial \phi}{\partial y}\left(\mathbf{r}, t^{\prime}\right), \\
2 v_{z}(\mathbf{r}, t) & =z \frac{\partial^{2} \phi(\mathbf{r}, t)}{\partial z^{2}}-\int_{-\infty}^{t} d t^{\prime} \kappa_{2}\left(t-t^{\prime}\right) \frac{\partial \phi}{\partial z}\left(\mathbf{r}, t^{\prime}\right),
\end{aligned}
$$

where $\kappa_{2}(t)$ is defined by

$$
\hat{\kappa}_{2}(\omega)=\frac{1}{2}(1+\hat{\kappa}(\omega))=2(1-\hat{\nu}(\omega))
$$

We also give the stress components $\sigma_{x z}, \sigma_{y z}$, and $\sigma_{z z}$ :

$$
\sigma_{x z}=z \frac{\partial^{3} \phi}{\partial x \partial z^{2}}, \quad \sigma_{y z}=z \frac{\partial^{3} \phi}{\partial y \partial z^{2}}, \quad \sigma_{z z}=z \frac{\partial^{3} \phi}{\partial z^{3}}-\frac{\partial^{2} \phi}{\partial z^{2}}
$$

These are independent of the material constants. The remaining components do depend on Poisson's ratio, however, in the elastic case and therefore will have hereditary integrals as in (3.6). We assume that all quantities vanish at infinity and that the boundary regions of interest are in the plane $z=0$. The tangential stresses are all zero on the plane $z=0$. Thus, they are specified everywhere, and $B_{u}^{(1)}\left(t^{\prime}\right)$ and $B_{u}^{(2)}\left(t^{\prime}\right)$ are empty for all $t^{\prime}$. We drop the superscript on $B_{u}^{(3)}\left(t^{\prime}\right)$ and $B_{\sigma}^{(3)}\left(t^{\prime}\right)$.

Consider the case where $B_{u}(t) \subseteq B_{u}\left(t^{\prime}\right), t^{\prime} \leq t$. On the boundary $z=0$, the last equation of (3.6) on $B_{u}(t)$ can be written as

$$
\int_{-\infty}^{t} d t^{\prime} l\left(t-t^{\prime}\right) u_{z}\left(\mathbf{r}, t^{\prime}\right)=-\frac{\partial \phi}{\partial z}(\mathbf{r}, t), \quad \mathbf{r} \in B_{u}(t),
$$

where $l(t)$ is defined by the requirement that its Fourier transform is given by

$$
\hat{l}(\omega)=\frac{4 \hat{\mu}(\omega)}{1+\hat{\kappa}(\omega)}=\frac{\hat{\mu}(\omega)}{1-\hat{\nu}(\omega)} .
$$

It is causal in the sense that $l(t)=0$ for $t<0$ by virtue of the argument given in [9].

The point is that if $B_{u}(t)$ is contained in $B_{u}\left(t^{\prime}\right), t^{\prime} \leq t$, then the hereditary integral on the left of (3.9) is given in terms of $d_{i}\left(\mathbf{r}, t^{\prime}\right), t^{\prime} \leq t$, on $B_{u}(t)$. The stress boundary condition is

$$
\sigma_{z z}(\mathbf{r}, t)=-\frac{\partial^{2} \phi}{\partial z^{2}}(\mathbf{r}, t)=c_{z}(\mathbf{r}, t), \quad \mathbf{r} \in B_{\sigma}(t) .
$$

We solve the problem by finding the harmonic function obeying these boundary conditions. This is also the solution to the elastic problem with the same specified stress and with $(\mu /(1-\nu))$ times the normal displacement given by the integral on the left of (3.9), where $\mu$ is the elastic shear modulus and $\nu$ is the elastic Poisson's ratio. The three stresses in (3.8) will be given by the elastic form of the solution for these boundary quantities, since (3.8) is independent of the material parameters. This does not apply to the other stresses, nor to the quantities $v_{x}(\mathbf{r}, t)$ etc., as given by 
(3.6). The other stresses contain hereditary integrals over $\phi\left(\mathbf{r}, t^{\prime}\right), t^{\prime} \leq t$. However, $\phi\left(\mathbf{r}, t^{\prime}\right)$ at times earlier than $t$ is not necessarily known. The argument above relies on special circumstances prevailing at time $t$. Therefore, the stresses $\sigma_{x x}, \sigma_{y y}, \sigma_{x y}$ are not necessarily calculable, by virtue of this principle. The same applies to the quantities on the left of (3.6). But even if these were known, it would not necessarily be possible to deduce the displacements, since once again the functions $v_{x}\left(\mathbf{r}, t^{\prime}\right)$ may not be known for $t^{\prime} \leq t$.

If the specified displacement is zero on $B_{u}(t)$ then, on this region, the left-hand side of (3.9) is zero. It follows that the stresses $\sigma_{x z}, \sigma_{y z}, \sigma_{z z}$ are identical in form to these for the corresponding elastic problem. These circumstances apply in the case of mode I crack problems.

In the case of plane problems, a more general result may be obtained, which we briefly note. In this case, the Papkovich-Neuber relations are replaced by the Kolosov-Muskhelishvili equations, which, for crack or half-plane problems, may be written in terms of one complex potential. The equations giving the stresses in terms of this potential do not depend explicitly on the material parameters. If, in the displacement equation, all the dependence on the material parameters can be grouped to form one hereditary integral over the displacement, in the statement of the boundary conditions, then the argument outlined above for the three-dimensional case goes through and all the stresses are given by the elastic form in terms of the known boundary quantities. This is always possible for crack problems (even in the presence of mode II shear stresses on the crack face) provided that the displacement difference, or the gap, across the axis containing the crack is used, rather than the displacement itself. A nontrivial instance of the generalized partial correspondence principle for a crack problem (mode I) has been given in [11]. See also [12], which incorporates mode II shear stresses on the crack face.

Now consider the case where $B_{\sigma}(t) \subseteq B_{\sigma}\left(t^{\prime}\right)$ for all $t^{\prime} \leq t$. If one writes down the above equations in terms of the harmonic functions,

$$
\chi(\mathbf{r}, t)=\int_{-\infty}^{t} d t^{\prime} k\left(t-t^{\prime}\right) \phi\left(\mathbf{r}, t^{\prime}\right),
$$

where $k(t)$ is the inverse of $l(t)$ in the sense that they are related by an equation similar to (2.10). Then (3.9) and (3.11) in particular become

$$
\begin{gathered}
u_{z}(\mathbf{r}, t)=-\frac{\partial}{\partial z} \chi(\mathbf{r}, t), \quad \mathbf{r} \in B_{u}(t), \\
\int_{-\infty}^{t} d t^{\prime} k\left(t-t^{\prime}\right) c_{z}\left(\mathbf{r}, t^{\prime}\right)=-\frac{\partial^{2}}{\partial z^{2}} \chi(\mathbf{r}, t), \quad \mathbf{r} \in B_{\sigma}(t),
\end{gathered}
$$

where the left-hand side of the second relation is known. The solution is a harmonic function obeying these boundary conditions and is the same as the elastic solution with these boundary conditions. The quantity $\chi(\mathbf{r}, t)$ is given at time $t$ and not necessarily at earlier times. However, if we operate on each equation of (3.6) with $\gamma(t)$ in a convolution sense to obtain diaplacements on the left-hand side, and also replace $\phi(\mathbf{r}, t)$ by its expression in terms of $\chi(\mathbf{r}, t)$, it is clear that the displacements will in general depend on hereditary integrals over $\chi(\mathbf{r}, t)$ and thus are not necessarily 
known. The exception is $u_{z}(\mathbf{r}, t)$ for $\mathbf{r}$ in the plane $z=0$. Outside the region $B_{u}(t)$ (where it is known anyway) it will still be given by the first equation of (3.13) and therefore by the elastic form in terms of the given boundary quantities, namely those on the left of (3.13). If $c_{z}\left(\mathbf{r}, t^{\prime}\right)$ is zero for all $t^{\prime} \leq t$ then the hereditary integral playing the part of the specified stress is zero and $u_{z}(\mathbf{r}, t), \mathbf{r} \in B_{\sigma}(t)$, will be given by precisely the elastic form for the same specified displacement on $B_{u}(t)$ and zero stress on $B_{\sigma}(t)$. This situation would prevail in the case of contact problems. An observation by Ting [13] is related to this result.

Equation (3.1) can be generalized to apply to aging viscoelastic materials (see [14]). Furthermore, the above arguments do not rely in an essential way on the convolution form of the hereditary integrals. Fourier transforms are used merely for convenient definition of various functions. It follows that the results of this paper may be adapted to the case of aging materials (see [7]).

4. Example: a contact problem. We now present an example to illustrate the utility of the partial correspondence principles given above. We shall work in terms of circular cylindrical coordinates $(\rho, \theta, z)$. A smooth rigid spherical indentor of radius $R$ is pressed into an isotropic and homogeneous viscoelastic half-space occupying the region $z>0$ by a normal time-dependent load. Body force and temperature fields are absent and all field quantities are functions of $(\rho, z, t)$ and independent of $\theta$. Provided the radius of the contact area remains small compared to that of the sphere, the boundary conditions may be written in the form

$$
\begin{array}{rlrl}
u_{z}(\rho, 0, t) & =D(t)-\rho^{2} /(2 R), & & 0 \leq \rho \leq a(t), \\
\sigma_{z z}(\rho, 0, t) & =0, \quad \rho>a(t), & \\
\sigma_{\rho z}(\rho, 0, t) & =\sigma_{\theta z}(\rho, 0, t)=0, & & \rho \geq 0,
\end{array}
$$

and the conditions at infinity are

$$
\sigma_{i j}(\rho, z, t) \rightarrow 0 \text { as }\left(\rho^{2}+z^{2}\right) \rightarrow \infty, \text { all }(i, j) .
$$

Here $D(t)$ specifies the time-dependent depth of penetration of the tip of the indentor into the half-space while $a(t)$ is the radius of the circular area of contact.

First we consider the case when $a\left(t^{\prime}\right) \geq a(t), t^{\prime} \leq t$. In the notation of previous sections this corresponds to the circumstance that $B_{\sigma}^{(3)}\left(t^{\prime}\right) \subseteq B_{\sigma}^{(3)}(t), t^{\prime} \leq t ; B_{\sigma}^{(i)}(t) \equiv$ $B, i=1,2 ; c_{i}(\mathbf{r}, t) \equiv 0, i=1,2,3$, and $d_{3}(\mathbf{r}, t)=D(t)-\rho^{2} /(2 R)$. By combining (4.1a) with (3.9) we find that for this problem

$$
\int_{-\infty}^{t} d t^{\prime} l\left(t-t^{\prime}\right) D\left(t^{\prime}\right)-\frac{\rho^{2} l_{e}}{2 R}=-\frac{\partial \phi}{\partial z}(\rho, 0, t), \quad 0 \leq \rho \leq a(t)
$$

where

$$
l_{e}=\int_{0}^{\infty} d t^{\prime} l\left(t^{\prime}\right)
$$

while, by using (4.1b), Eq. (3.11) becomes

$$
0=-\frac{\partial^{2} \varphi}{\partial z^{2}}(\rho, 0, t), \quad \rho>a(t) .
$$


Using the fact that (3.8) is independent of material properties and the elastic solution of the contact problem governed by (4.3), (4.5) (see Sneddon [15]), we find that for the viscoelastic problem under consideration

$$
\sigma_{z z}(\rho, 0, t)=\frac{4 l_{e}}{\pi R}\left(a^{2}(t)-\rho^{2}\right)^{1 / 2}, \quad 0 \leq \rho \leq a(t),
$$

where $a(t)$ is related to the history of indentation by

$$
\frac{1}{l_{e}} \int_{-\infty}^{t} d t^{\prime} l\left(t-t^{\prime}\right) D\left(t^{\prime}\right)=\frac{a^{2}(t)}{R}=D_{e}(t)
$$

It follows from (4.6) that the total load acting in the indentor is

$$
W(t)=\frac{8 l_{e}}{3 R} a^{3}(t)=W_{e}(t)
$$

Here and later " $e$ " is used to indicate elastic quantities. Further, by considering the particular circumstance that $a\left(t^{\prime \prime}\right)=a(t), t^{\prime} \leq t^{\prime \prime} \leq t$, and leaving $a\left(t^{\prime \prime}\right), t^{\prime \prime}<t^{\prime}$, unaltered we conclude that

$$
\sigma_{z z}(\rho, 0, t) \leq \sigma_{z z}\left(\rho, 0, t^{\prime}\right), \quad W(t) \leq W\left(t^{\prime}\right), t^{\prime} \leq t,
$$

because to achieve such a sudden reduction in contact area at $t^{\prime}$ a decrease in applied load will be required if $a\left(t^{\prime}\right)<a(t)$, while if $a\left(t^{\prime}\right)=a(t)$ the results (4.6), (4.8) apply at time $t^{\prime}$ and (4.9) is an equality. Thus the applied loads achieve a minimum simultaneously with the contact area.

In the particular circumstance that $a\left(t^{\prime}\right),-\infty<t^{\prime} \leq t$, is constant or monotonic the normal surface displacement outside the contact area may be calculated by inverting (see Sneddon [15])

$$
\begin{aligned}
\int_{-\infty}^{t} d t^{\prime} l(t & \left.-t^{\prime}\right) u_{z}\left(\rho, 0, t^{\prime}\right) \\
& = \begin{cases}\frac{l_{e}}{R}\left(a^{2}(t)-\frac{\rho^{2}}{2}\right), \quad \rho \leq a(t), \\
\frac{l_{e}}{\pi R}\left\{\left(2 a^{2}(t)-\rho^{2}\right) \sin ^{-1}\left(\frac{a(t)}{\rho}\right)+\sqrt{\rho^{2}-a^{2}(t)}\right\}, & \rho>a(t) .\end{cases}
\end{aligned}
$$

We now apply an argument similar to that developed after (4.9) to show that

$$
D_{e}(t)=\min _{t^{\prime} \leq t} \frac{1}{l_{e}} \int_{-\infty}^{t^{\prime}} d t^{\prime \prime} l\left(t^{\prime}-t^{\prime \prime}\right) D\left(t^{\prime \prime}\right)
$$

If $a\left(t^{\prime}\right)>a(t)$, consider a sudden decrease in contact area to $a(t)$ at this time, as defined before (4.9). Such a decrease will entail a reduction in the indentation. Furthermore, after this decrease, it follows from (4.7) that

$$
\frac{1}{l_{e}} \int_{-\infty}^{t^{\prime}} d t^{\prime \prime} l\left(t^{\prime}-t^{\prime \prime}\right) D\left(t^{\prime \prime}\right)=D_{e}\left(t^{\prime}\right)=D_{e}(t)
$$

Also, $l(t)$ can be written as the sum of a singular and a slowly varying term, in the same manner as $\mu(t)$, given by (1.3). The phenomenon of relaxation leads us to conclude that the slowly varying part is nonpositive. It follows, on using these observations in (4.12), that $D_{a}\left(t^{\prime}\right) \geq D_{e}(t)$, where $D_{a}\left(t^{\prime}\right)$ is the indentation after the sudden decrease in contact area. Thus $D\left(t^{\prime}\right)>D_{a}\left(t^{\prime}\right) \geq D_{e}(t)$. If $a\left(t^{\prime}\right)=a(t)$ the 
same argument goes through, omitting the portions related to the sudden decrease in contact area. Thus

$$
D\left(t^{\prime}\right) \geq D_{e}(t), \quad-\infty<t^{\prime} \leq t
$$

We deduce that

$$
\frac{1}{l_{e}} \int_{-\infty}^{t^{\prime}} d t^{\prime \prime} l\left(t^{\prime}-t^{\prime \prime}\right)\left[D\left(t^{\prime \prime}\right)-D_{e}(t)\right] \geq 0
$$

since operating with $l\left(t^{\prime}-t^{\prime \prime}\right)$ cannot cause a change of sign. This is clear in the case of a material with unique Poisson's ratio, since $l(t)$ reduces to $\mu(t) /(1-\nu)$ and $\mu(t)$ certainly has this property. If it did not, then we see from (2.1) that a given shear stress could correspond to a history of shear strain of the opposite sign. More generally, we can argue on the basis of the generalized Boussinesq equation relating surface displacement and stress (see [17]) which contains a hereditary integral over displacement with $l\left(t-t^{\prime}\right)$ as the kernel. If $l\left(t-t^{\prime}\right)$ could cause a change of sign, then a positive surface pressure could be associated with a history of negative surface displacement. Equation (4.11) follows from (4.14). This result will be used later.

Next consider the case when $a\left(t^{\prime}\right) \leq a(t), t^{\prime} \leq t$. This corresponds to the circumstances that $B_{\sigma}^{(3)}\left(t^{\prime}\right) \supseteq B_{\sigma}^{(3)}(t), t^{\prime} \leq t ; B_{\sigma}^{(i)}(t) \equiv B, i=1,2$; and $c_{i}$ and $d_{3}$ are constrained as in the previous case. By combining $(4.1 \mathrm{a}, \mathrm{b})$ with $(3.13)$ we find that

$$
\begin{array}{rlrl}
D(t)-\rho^{2} /(2 R) & =-\frac{\partial \chi}{\partial z}(\rho, 0, t), & 0 \leq \rho \leq a(t), \\
0 & =-\frac{\partial^{2} \chi}{\partial z^{2}}(\rho, 0, t), \quad \rho>a(t) .
\end{array}
$$

The displacement $u_{z}(\rho, 0, t)$, is the same as in the elastic case by virtue of the argument after (3.13). We therefore have (Sneddon [15])

$$
u_{z}(\rho, 0, t)=\frac{1}{\pi R}\left\{\left(2 a^{2}(t)-\rho^{2}\right) \sin ^{-1}\left(\frac{a(t)}{\rho}\right)+\sqrt{\rho^{2}-a^{2}(t)}\right\}, \quad \rho \geq a(t),
$$

where $a(t)$ is related to $D(t)$ by

$$
D(t)=\frac{a^{2}(t)}{R}=D_{e}(t)
$$

At the same time

$$
\int_{-\infty}^{t} d t^{\prime} k\left(t-t^{\prime}\right) \sigma_{z z}\left(\rho, 0, t^{\prime}\right)=\frac{4}{\pi R}\left(a^{2}(t)-\rho^{2}\right)^{1 / 2}, \quad 0 \leq \rho \leq a(t) .
$$

Strictly (4.6) and (4.16) are manifestations of the generalized partial correspondence principle. Equations (4.7) and (4.17) are further relations which follow from known results in the elastic case and the formal analogy between (4.3), (4.5), (4.15), and the corresponding elastic problem. As before, consideration of the case when $a\left(t^{\prime \prime}\right)=$ $a(t), t^{\prime} \leq t^{\prime \prime} \leq t$, with $a\left(t^{\prime \prime}\right), t^{\prime \prime}<t$, unaltered implies that

$$
u_{z}(\rho, 0, t) \geq u_{z}\left(\rho, 0, t^{\prime}\right), \quad D(t) \geq D\left(t^{\prime}\right), \quad t^{\prime} \leq t .
$$

so that indentation is maximum at the same time as contact area.

Integration of (4.18) yields

$$
\frac{1}{k_{e}} \int_{-\infty}^{t} d t^{\prime} k\left(t-t^{\prime}\right) W\left(t^{\prime}\right)=W_{e}(t)
$$


where $k_{e} l_{e}=1$ and $W_{e}(t)$ is given by (4.8). In the particular case when $a\left(t^{\prime}\right),-\infty<$ $t^{\prime} \leq t$, is constant or monotone increasing, (4.20) is true for all time and may be inverted to express $W(t)$ in terms of the history of $W_{e}(t)$. Using essentially a similar argument to that yielding (4.11), one may show that

$$
W_{e}(t)=\max _{t^{\prime} \leq t} \frac{1}{k_{e}} \int_{-\infty}^{t^{\prime}} d t^{\prime \prime} k\left(t^{\prime}-t^{\prime \prime}\right) W\left(t^{\prime \prime}\right)
$$

provided $a\left(t^{\prime}\right) \leq a(t),-\infty<t^{\prime} \leq t$.

The forms of the above equations appropriate to a material with a unique Poisson's ratio $\nu$ may be obtained by replacing $l(t)$ by $\mu(t) /(1-\nu)$ as noted earlier, and $k(t)$ by $(1-\nu) \gamma(t)$. We now confine the discussion to materials of this kind.

Consider a strain-controlled history where the indentation is given by

$$
D\left(t^{\prime}\right)=A\left(d-\cos \left(\omega t^{\prime}\right)\right), \quad \text { for all } t^{\prime},
$$

and steady-state conditions are assumed to be established. It is further assumed that

$$
d \geq|\hat{\mu}(\omega)| / \hat{\mu}(0)
$$

where $\hat{\mu}(\omega)$ is the shear complex modulus of the material. Equation (4.7) becomes

$$
A\left\{d-(|\hat{\mu}(\omega)| / \hat{\mu}(0) \cos (\omega t+\varphi(\omega))\}=D_{\mathcal{C}}(t),\right.
$$

where $\varphi(\omega)$ is the loss angle of the viscoelastic material (see [7], for example). At times $t^{\prime} \leq t$, the left-hand side of (4.24) is equal to the right-hand side of (4.11) and we deduce that the special times $t$ of minimum contact area are given by

$$
t=(2 n \pi-\phi(\omega)) / \omega,
$$

where $n$ is any integer. These times precede the times of minimum indentation by $\varphi(\omega) / \omega$. The minimum value of the contact area may be deduced from (4.7) and

$$
D_{e}(t)=A\{d-(|\hat{\mu}(\omega)| / \hat{\mu}(0))\} .
$$

Equations (4.17), (4.19) give that the maximum value of $D_{e}(t)$ and of $a(t)$ occurs at the same time as that of $D(t)$. Also, from (4.22)

$$
\begin{aligned}
t & =2 \pi\left(n+\frac{1}{2}\right) / \omega, \\
D_{e}(t) & =D(t)=A(d+1),
\end{aligned}
$$

where $n$ is any integer. The maximum contact area can be determined from the second relation.

Finally, consider the stress-controlled case where

$$
W\left(t^{\prime}\right)=K\left(d-\cos \left(\omega t^{\prime}\right)\right), \quad d \geq 1 .
$$

Equation (4.20) gives that, at the time of maximum contact area,

$$
W_{c}(t)=K(d-(\hat{\mu}(0) /|\hat{\mu}(\omega)|) \cos (\omega t-\varphi(\omega))),
$$

where $W_{e}(t)$ is related to $a(t)$ by (4.8). From (4.21) we deduce that the times of maximum contact area are given by

$$
t=\left[\varphi(\omega)+2 \pi\left(n+\frac{1}{2}\right)\right] / \omega
$$


where $n$ is any integer. These are later than the times of maximum load by $\varphi(\omega) / \omega$. From (4.28) and (4.9) we deduce that the occurrences of minimum load and minimum contact area are simultaneous, both at times $2 n \pi / \omega$ for any integer $n$. The value of the minimum contact area follows from (4.8) and the relation

$$
W_{e}(t)=K(d-1) \text {, }
$$

where $t$ is one of these times.

Equations (4.7), (4.8), (4.17), (4.18), and (4.30) are generalizations of results demonstrated for periodic loading of a punch on a standard linear solid by Golden and Graham [16] and Graham and Golden [17]; see also [7]. These results can be generalized without difficulty to the case of a general punch shape.

5. Conclusions. Thus, in a mixed boundary value problem for a viscoelastic material with unique Poisson's ratio, where the boundary regions may be different for different components of displacement and stress, if $B_{u}^{(i)}(t)$ is such that $B_{u}^{(i)}\left(t^{\prime}\right) \subseteq B_{u}^{(i)}(t)$, $i=1,2,3$, for all $t^{\prime} \leq t$, then at time $t$ the displacement everywhere is given by the same form as for the elastic problem with the same boundary regions and the same specified displacements but where the specified stresses are replaced by hereditary integrals over these stresses. By virtue of the assumption on $B_{u}^{(i)}(t)$, these integrals are known. If, where the stresses are specified, they vanish for all $t^{\prime} \leq t$ then these hereditary integrals are zero, and the displacements are given by precisely the elastic form. The most immediate example of this is that of frictionless contact problems. Note that the assumption on $B_{u}^{(i)}(t)$ imposes no constraint on the history of this region except that specified. In particular, it is far more general than the assumption that $B_{u}^{(i)}(t)$ is monotonically increasing.

If, on the other hand, we have that at a given time $B_{\sigma}^{(i)}\left(t^{\prime}\right) \subseteq B_{\sigma}^{(i)}(t)$, for all $t^{\prime} \leq t$, then the stresses at time $t$ will be given by the stresses of the elastic problem with the same boundary regions and the same specified stresses, but where the specified displacements are replaced by known hereditary integrals of the originally prescribed displacements. If the displacements are always prescribed to be zero, the stresses are identically those of the elastic problem. This happens in crack problems. For example, in cracks under mode I displacement, only the normal displacement is important and this is zero off the crack face. In fact, a nontrivial instance of the generalized partial correspondence principle has been demonstrated for such cracks in [11]. In the case of more general crack problems, if they can be phrased so that the gap on the $x$-axis (along which the crack is lying) is the effective boundary quantity rather than displacement itself, then the gap is always zero off the crack face (see [12]).

There are restricted conditions under which it is not necessary to assume that the material has a unique Poisson's ratio. A particular problem class of this kind is discussed in Sec. 3 and an example is worked out in Sec. 4. The problems discussed in $[11,12,13,16,17]$ are all members of the class. For these problems, the statement of the principle is altered and usually restricted to special circumstances. Though presented for the nonaging case, the general results also apply to aging viscoelastic materials. 
Acknowledgment. This work was supported by funds provided by the Natural Sciences and Engineering Research Council of Canada.

\section{REFERENCES}

[1] R. M. Christensen, Theory of viscoelasticity, Academic Press, New York, 1971

[2] G. A. C. Graham, On the solution of mixed boundary value problems that involve time-dependent boundary regions for viscoelastic bodies with temperature dependent properties, Arch. Mech. Stos. $\mathbf{5}$, $771-785$ (1967)

[3] G. A. C. Graham, The correspondence principle of linear viscoelasticity theory for mixed boundary value problems involving time-dependent boundary regions, Quart Appl. Math. 26, 167-174 (1968)

[4] T. C. T. Ting, A mixed boundary value problem in viscoelasticity with time-dependent boundary regions, Proc. of the Eleventh Midwestern Mechanics Conference, H. J. Weiss, D. F. Young, W. F. Riley, and T. R. Rogge (Editors), Iowa University Press, 591-599, 1969

[5] G. A. C. Graham and G. C. W. Sabin, The correspondence principle of linear viscoelasticity for problems that involve time-dependent regions, Internat J. Engng. Sci. 11, 123-140 (1973)

[6] G. A. C. Graham, The solution of mixed boundary value problems that involve time-dependent boundary regions for viscoelastic materials with one relaxation function, Acta Mech. 8, 188-204 (1969)

[7] J. M. Golden and G. A. C. Graham, Boundary value problems in linear viscoelasticity, Springer-Verlag, Berlin, 1988

[8] M. E. Gurtin and E. Sternberg, On the linear theory of viscoelasticity, Arch. Rat. Mech. Anal. 11, 291-356 (1962)

[9] J. M. Golden, Causality and viscoelastic boundary value problems, Internat J. Engng. Sci. 24, 11411149 (1986)

[10] A. E. Green and W. Zerna, Theoretical elasticity, Clarendon Press, Oxford, 1968

[11] G. A. C. Graham and G. C. W. Sabin, The opening and closing of a growing crack in a linear viscoelastic body that is subject to alternating tensile and compressive loads, Internat $\mathrm{J}$. Fracture 14, 639-649 (1978)

[12] J. M. Golden and G. A. C. Graham, Energy balance criteria for viscoelastic fracture, submitted for publication

[13] T. C. T. Ting, Contact problems in the linear theory of viscoelasticity, J. Appl. Mech. 35, 248-254 (1968)

[14] G. A. C. Graham and F. M. Williams, Boundary value problems for time-dependent regions in aging viscoelasticity, Utilitas Math. 2, 291-303 (1972)

[15] I. N. Sneddon, The relation between load and penetration in the axisymmetric Boussinesq problem for a punch of arbitrary profile, Internat J. Engng. Sci. 3, 47-57 (1965)

[16] J. M. Golden and G. A. C. Graham, The steady-state plane normal viscoelastic contact problem, Internat J. Engng. Sci. 25, 227-291 (1987)

[17] G. A. C. Graham and J. M. Golden, The three-dimensional steady-state viscoelastic indentation problem, Internat J. Engng. Sci. 26, 121-126 (1988) 